\title{
Evidências sobre o suporte durante o trabalho de parto/parto: uma revisão da literatura
}

\author{
Evidence on support during labor \\ and delivery: a literature review
}

\footnotetext{
1 Faculdade de Ciências Médicas, Universidade Estadual de Campinas, Campinas, Brasil.

2 Departamento de Enfermagem, Universidade Federal de Santa Catarina, Florianópolis, Brasil.

3 Centro de Pesquisas Materno-Infantis de Campinas, Campinas, Brasil

Correspondência O. M. Brüggemann Departamento de Enfermagem, Universidade Federal de Santa Catarina. Rua Santo Inácio de Loyola 64, apto. 103, Florianópolis, SC 88015-330, Brasil. odalea@nfr.ufsc.br
}

\begin{abstract}
The effects of support for women during labor and delivery provided by health professionals, lay women, and doulas on the maternal and neonatal outcomes have been evaluated through randomized clinical trials, meta-analyses, and systematic reviews. This article presents a review of these studies, focusing on the principal characteristics, support provider, simultaneous presence of the woman's spouse and/or family members during labor and delivery and the outcomes. The analysis included studies published from 1980 to 2004 which explicitly approached these aspects. In general, the results of such support were favorable, highlighting a reduction in the cesarean rate, analgesia/ medication for pain relief, duration of labor, and utilization of oxytocin and an increase in maternal satisfaction with the experience. The benefits were greater when the support provider was not a health professional. The available studies did not evaluate the specific companion chosen by the woman as a support provider, which constitutes a gap in the knowledge that should be filled by future research.
\end{abstract}

\section{Obstetric Labor; Parturition; Perinatal Care}

Odaléa Maria Brüggemann 1,2

Mary Angela Parpinelli 1

Maria José Duarte Osis 1,3

\section{Introdução}

Historicamente, o acompanhamento do trabalho de parto e parto ocorria no ambiente domiciliar, no qual a mulher era assistida por outra mulher, geralmente uma parteira ou uma "aparadeira” de sua confiança, e apoiada pelos seus familiares. No século XX, mais expressivamente depois da Segunda Guerra Mundial, em nome da redução das elevadas taxas de mortalidade materna e infantil ocorre a institucionalização do parto, passando do domicilio para o hospital, e conseqüentemente a sua medicalização 1 .

A institucionalização do parto foi um fator determinante para afastar a família e a rede social do processo do nascimento, uma vez que a estrutura física e as rotinas hospitalares foram planejadas para atender as necessidades dos profissionais de saúde, e não das parturientes 2,3 . Assim, a maioria das mulheres passou a permanecer internada em sala de pré-parto coletivo, com pouca ou nenhuma privacidade, assistidas com práticas baseadas em normas e rotinas que as tornaram passivas e impediram ou impossibilitaram a presença de uma pessoa de seu convívio social para apoiá-las.

Os procedimentos de internação em maternidade acontecem como um verdadeiro cerimonial, um rito de passagem, repleto de rotinas e normas de comportamento que são ditadas pela instituição ${ }^{2}$. De maneira geral, existe uma contradição entre o que prescreve a ciên- 
cia e a maneira como as práticas se organizam. Esta irracionalidade tem intrigado muitos estudiosos que lidam com a medicina perinatal, os movimentos sociais, além dos antropólogos, compondo o que se chama hoje de "antropologia do parto" 3 .

Nas últimas décadas, em hospitais do mundo, o suporte à mulher durante o trabalho de parto tornou-se mais uma exceção do que uma rotina. O interesse sobre o retorno desse apoio vem acontecendo como uma das estratégias de humanização do nascimento 4 .

A presença de um acompanhante durante o trabalho de parto e parto difere de acordo com o contexto social, a política de saúde do país e sua legislação, mas principalmente de acordo com a filosofia da maternidade. Assim, em alguns locais, essa presença é estimulada e permitida, em outros não é permitida ou há restrições 5 .

No Brasil, algumas maternidades estão adequando a sua área física para possibilitar a permanência de um acompanhante, escolhido pela parturiente, sem comprometer a privacidade das demais parturientes, e até mesmo implantando o modelo conhecido como PPP (Pré-parto, Parto e Puerpério), que possibilita que a assistência nos períodos clínicos do parto seja realizada no mesmo local, o que proporciona conforto e individualidade 6,7 .

O processo de mudança no panorama brasileiro foi estimulado por diversos acontecimentos, entre eles a Conferência sobre Tecnologia Apropriada para o Nascimento e Parto, que ocorreu em Fortaleza, em 1985, na qual a Organização Mundial da Saúde (OMS) recomendou o livre acesso de um acompanhante, escolhido pela parturiente, no parto e puerpério. Essa recomendação, entre outras, foi baseada na revisão do conhecimento sobre o uso de tecnologia de nascimento que indica a contribuição dessa prática para o bem-estar da parturiente 8 .

As evidências científicas sobre os benefícios do acompanhante para a melhoria dos indicadores de saúde e do bem-estar da mãe e do recém-nascido inspiraram a Rede de Humanização do Nascimento (REHUNA) a iniciar uma campanha pelo direito da parturiente a um acompanhante de sua escolha. Essa campanha foi lançada em maio de 2000 e contou com o apoio da Rede Nacional Feminista de Saúde, Direitos Sexuais e Direitos Reprodutivos, da Associação Brasileira de Obstetrizes e Enfermeiras Obstetras, e da União dos Movimentos Populares de Saúde de São Paulo 9 .
Essa campanha se ampliou e foi apoiada pelos profissionais de saúde das maternidades que já permitiam a presença do acompanhante. A senadora Ideli Salvati (PT/SC), com suporte técnico dos pesquisadores do Núcleo Interdisciplinar de Pesquisa sobre Parto e Nascimento da Universidade Federal de Santa Catarina, elaborou o projeto que resultou na Lei $n$. 12.333, de 12 de março de 2002, assegurando o acompanhamento no parto em todos os hospitais públicos e conveniados naquele Estado. Também apresentou ao Senado Federal projeto similar para que este benefício fosse para todas as mulheres do país 10 .

Como resultado de toda essa mobilização, foi aprovada pelo Congresso Nacional e sancionada pelo Presidente da República a Lei n. 11.108, de 7 de abril de 2005, que obriga os serviços de saúde do Sistema Único de Saúde (SUS), da rede própria ou conveniada, a permitirem a presença de um acompanhante escolhido pela parturiente durante todo o período de trabalho de parto, parto e pós-parto imediato 11.

De maneira geral, os profissionais de saúde possuem receio e idéias pré-concebidas negativas sobre a presença do acompanhante no contexto do nascimento 12,13. A necessidade de implementar a nova legislação oportuniza que esses profissionais vivenciem a experiência, e conseqüentemente possam identificar e compreender os aspectos que envolvem essa prática.

A presença de um acompanhante, mesmo que escolhido pela parturiente, por si só não pode ser considerado como sinônimo de suporte, entretanto, pode ser dado a ele condições e apoio para realizar essa atividade. $O$ suporte no trabalho de parto consiste na presença de uma pessoa que oferece conselhos, medidas de conforto físico e emocional, e outras formas de ajuda para a parturiente durante o trabalho de parto e parto 14,15 .

A OMS recomenda o respeito à escolha da mulher sobre seus acompanhantes durante o trabalho de parto. A parturiente deve ser acompanhada por pessoas em que confia e com quem se sinta à vontade 16. Na literatura, o conceito de acompanhante tem sido utilizado para descrever o suporte por diferentes pessoas que possuem características muito distintas, de acordo com o contexto assistencial envolvido, podendo ser profissionais (enfermeira, parteira), companheiro/familiar ou amiga da parturiente, doula e mulher leiga designada para tal função.

Na América do Norte, as mulheres com um treinamento especial para dar suporte são co- 
nhecidas como doulas (do grego = mulher que serve), mas também são chamadas de acompanhantes de trabalho de parto, acompanhantes de parto, assistentes de trabalho de parto ou parto ${ }^{4}$. Essas diversas denominações geram na literatura e na prática assistencial uma certa ambigüidade conceitual. Assim, o termo doula é utilizado, de maneira geral, para denominar mulheres que dão suporte à parturiente, mas sem muitas vezes levar em conta a sua formação ou nível de treinamento.

Apenas estudos observacionais têm buscado compreender a utilidade e a forma de suporte provido por familiares, pelo cônjuge e amigos da parturiente 14. A parturiente percebe a presença desse acompanhante como de grande ajuda, especialmente relacionada ao apoio emocional e conforto físico 17 . Dentre as atividades realizadas, o acompanhante permanece ao lado da parturiente segurando a sua mão, realiza massagens, auxilia no banho e na deambulação, e encoraja no período expulsivo 7,18. As atividades de suporte variam de acordo com as necessidades, desejos e cultura da mulher 14 .

Por outro lado, a avaliação dos efeitos do suporte dado à mulher durante o trabalho de parto/parto por profissionais de saúde, doulas e mulheres leigas tem sido foco de estudo em vários ensaios clínicos randomizados e revisões sistemáticas, tornando-os uma prática embasada nas evidências científicas. Este artigo tem como objetivo apresentar as principais características desses estudos, e se os seus resultados se associam ou não com o tipo de provedor de suporte e com a simultaneidade ou não da presença do companheiro/familiares da parturiente. Ao mesmo tempo, pretende-se identificar se a presença de acompanhantes/ familiares tem sido avaliada como uma forma de suporte ou se estão presentes somente para compartilhar a experiência.

\section{Sujeitos e métodos}

A Prática Baseada em Evidência é uma proposta assistencial e pedagógica fundamentada na epidemiologia clínica, bioestatística e informática em saúde, desenvolvida na década de 1980 na Universidade de McMaster, no Canadá. Ela pretende preencher a lacuna entre a pesquisa qualificada e a prática correta, por meio da busca de evidências que embasam as condutas e procedimentos. Este novo paradigma reconhece o valor da experiência clínica e da avaliação intuitiva, mas propõe que elas sejam alicerçadas em evidências. Dessa forma pode contribuir para limitar o autoritarismo no ensino e na prática assistencial, através do estimulo à iniciativa e criatividade 19.

O início desse movimento é atribuído ao epidemiologista Dr. Archie Cochrane. Na medicina perinatal, a primeira revisão sistemática de ensaios clínicos randomizados controlados foi publicada em 1989 por Chalmers et al. - Effetive Care in Pregnancy and Childbirth - e na forma eletrônica foi a Oxford Database of Perinatal Trials. Em 1992 foi desenvolvido o projeto conhecido como Cochrane Collaboration, que contempla as revisões de ensaios clínicos controlados na área de saúde 20.

Para a elaboração desta revisão foi realizada uma pesquisa nas bases de dados MEDLINE, LILACS, PubMed, SciELO e ISI Web of Science, entre os anos de 1980 e 2004, com base nas palavras-chave: suporte/apoio (support), acompanhante (companionship or companion), doula, trabalho de parto (labor) e parto (childbirth or delivery).

Os estudos selecionados foram classificados em duas categorias: os que apresentavam evidências grau I (ensaios clínicos randomizados) e os que sintetizavam as evidências (revisões sistemáticas e metanálises) sobre o suporte fornecido à mulher durante o trabalho de parto e parto. A partir dessa seleção, foram incluídos somente os ensaios clínicos randomizados que compararam um grupo com suporte e outro sem; que apresentavam informações sobre as parturientes, quem era o provedor de suporte, os resultados maternos/perinatais; e os que explicitavam se era permitida ou não a presença do companheiro/familiares da parturiente. E também foram incluídas as revisões sistemáticas e metanálises que avaliaram ensaios clínicos randomizados. Considerando esses critérios, o material analisado constou de dez ensaios clínicos randomizados (nove publicados na íntegra e um na forma de abstract), duas revisões sistemáticas e duas metanálises.

Constituído o conjunto de ensaios clínicos a serem analisados, identificaram-se as peculiaridades assistenciais das instituições em que foram desenvolvidos, as características do provedor de suporte, a simultaneidade ou não da presença do companheiro/familiares da parturiente, as variáveis sobre a evolução do trabalho de parto/parto, e os resultados maternos e perinatais. As revisões sistemáticas e metanálises foram utilizadas para consolidar alguns resultados e auxiliar na discussão dos mesmos.

Para este trabalho de revisão, considerouse o suporte durante o trabalho de parto como a presença de uma pessoa designada para desenvolver atividades de apoio, que contemplam os aspectos emocionais (encorajar, tranqüili- 
zar e estimular), medidas de conforto e orientações. Entendeu-se o termo doula como mulher designada para dar suporte à parturiente, que recebeu algum tipo de treinamento, não integrante do quadro de funcionários do hospital em que desempenhou este papel e que não possuía parentesco com a parturiente.

\section{Resultados}

Os resultados serão apresentados em dois eixos condutores. Inicialmente, serão ressaltados aspectos relacionados às características dos ensaios clínicos e, posteriormente, uma análise das variáveis avaliadas nesses estudos e os resultados maternos e perinatais.

\section{Características dos estudos}

Os estudos selecionados apresentam diferentes características no que se refere ao nível de desenvolvimento do país em que foram realizados, aos sujeitos, ao tipo de provedor de suporte, à presença ou não do companheiro ou familiares da parturiente. Essas características são evidenciadas na Tabela 1, conforme descrito a seguir.

Os primeiros ensaios clínicos para avaliar os efeitos do suporte à mulher durante o trabalho de parto/parto foram realizados na Guatemala, na década de 80 , tendo mulheres leigas, sem treinamento, como protagonistas dessa intervenção. Na década de 90, estudos semelhantes foram realizados na América do Norte com doulas. Nessas duas décadas e no início do século XXI também foram desenvolvidos estudos no Canadá, para avaliar o suporte provido por profissionais de saúde (parteiras leigas ou em treinamento, enfermeiras). Os efeitos do suporte provido por parente da parturiente, do sexo feminino, foi avaliado em apenas um estudo.

Outro aspecto importante é o nível de desenvolvimento dos países em que os estudos foram realizados, que os autores consideraram como diretamente relacionado às características dos hospitais envolvidos nas pesquisas, embora somente alguns explicitem as rotinas obstétricas presentes. O amplo uso da tecnologia disponível e o manejo ativo do trabalho de parto, típico de países desenvolvidos da América do Norte, são características que podem influenciar nos resultados. Os estudos de Sosa et al. 21 e Klaus et al. 22 foram realizados em hospitais que possuíam poucas intervenções tecnológicas, inclusive o monitoramento fetal eletrônico não estava disponível e o acompanhamento do trabalho de parto era realizado clini- camente. O ensaio clínico de Kennell et al. 23 pretendeu avaliar se os efeitos do suporte contínuo, encontrados nos estudos de Sosa et al. 21 e Klaus et al. 22 , poderiam ser similares em um hospital de ensino que possuía toda variedade de equipamentos, técnicas obstétricas modernas, manejo ativo do trabalho de parto e as parturientes eram mantidas em leitos de préparto para facilitar a rotina hospitalar.

Com relação aos sujeitos envolvidos nos ensaios clínicos, observa-se pouca variação em termos de paridade, apesar de diferir a terminologia. Na maioria das vezes, eram mulheres primíparas, de baixo risco e em início da fase ativa do trabalho de parto. Cabe destacar que no estudo de Hodnett et al. 24 participaram nulíparas e multíparas. Para o estudo de Kennell \& McGrath 25, este dado não estava disponível.

Quanto ao tipo de suporte, nos estudos em que era provido por doulas, observou-se uma diversidade em relação à sua formação e nível de treinamento. No ensaio clínico de Langer et al. 26, a maioria das doulas possuía formação profissional, pois eram enfermeiras aposentadas que foram treinadas e contratadas para realizar suporte emocional, medidas de conforto físico, fornecer informações sobre a evolução do trabalho de parto, comunicação social, assegurar contato imediato entre mãe e bebê após o nascimento, além de oferecer orientações sobre o aleitamento durante uma única e breve sessão pós-natal. No estudo de Kennell et al. 23 eram mulheres que receberam treinamento sobre técnicas de suporte, procedimentos obstétricos, evolução normal e distócica do trabalho de parto, e sobre as rotinas do hospital. Essas mulheres foram remuneradas pela sua atividade, que também incluía a transmissão de instruções médicas para a parturiente, quando necessário.

No estudo de Hofmeyer et al. 27, mulheres voluntárias, selecionadas na comunidade, que possuíam habilidade para expressar empatia, que não tinham experiência de práticas médicas ou de enfermagem, assumiram o papel de confortar, tranqüilizar e incentivar as parturientes. Nos estudos de Sosa et al. ${ }^{21}$ e Klaus et al. 22, o provedor de suporte foram mulheres leigas sem treinamento. Todas essas mulheres são classificadas como doulas nas metanálises de Zhang et al. 28 e Scott et al. 29.

O suporte fornecido por profissionais de saúde, especificamente por enfermeiras e parteiras leigas ou em treinamento, também foram avaliados, embora em poucos estudos. Recente artigo de revisão sobre os diferentes provedores de suporte, no período de 1980 a 2003, mostra que os ensaios clínicos randomizados sobre esse tipo de suporte se iniciaram no final 
Características dos ensaios clínicos randomizados.

\begin{tabular}{|c|c|c|c|c|c|}
\hline Ensaios clínicos & Ano & País & Sujeitos & Provedor de suporte & Familiares ou companheiros \\
\hline Sosa et al. 21 & 1980 & Guatemala & $\begin{array}{l}40 \text { primigrávidas, início do } \\
\text { trabalho de parto }(1-2 \mathrm{~cm}) \text {, } \\
\text { baixo risco }\end{array}$ & Mulher leiga sem treinamento & Não permitida presença \\
\hline Klaus et al. 22 & 1986 & Guatemala & $\begin{array}{l}465 \text { primigrávidas, gestação à } \\
\text { termo, dilatação cervical } \leq 3 \mathrm{~cm} \text {, } \\
\text { baixo risco }\end{array}$ & $\begin{array}{l}\text { Mulher leiga sem treinamento } \\
\text { obstétrico }\end{array}$ & Não permitida presença \\
\hline $\begin{array}{l}\text { Hodnett \& } \\
\text { Osborn } 33\end{array}$ & 1989 & Canadá & 103 primigrávidas de baixo risco & $\begin{array}{l}\text { Parteira leiga ou em início } \\
\text { de treinamento }\end{array}$ & $\begin{array}{l}\text { Permitida presença do } \\
\text { marido ou companheiro }\end{array}$ \\
\hline Hofmeyer et al. 27 & 1991 & África do Sul & $\begin{array}{l}189 \text { nulíparas em trabalho de } \\
\text { parto, dilatação cervical }<6 \mathrm{~cm} \text {, } \\
\text { sem complicações obstétricas }\end{array}$ & $\begin{array}{l}\text { Mulher voluntária da } \\
\text { comunidade sem experiência } \\
\text { médica ou de enfermagem }\end{array}$ & Não permitida presença \\
\hline Kennell et al. 23 & 1991 & Estados Unidos & $\begin{array}{l}616 \text { nulíparas na fase do } \\
\text { trabalho de parto ativa, } \\
\text { dilatação cervical } 3-4 \mathrm{~cm}\end{array}$ & Doula (mulher treinada) & Não permitida a presença \\
\hline $\begin{array}{l}\text { Kennell \& } \\
\text { McGrath 25* }\end{array}$ & 1993 & Estados Unidos & 570 parturientes & Doula & Não permitida a presença \\
\hline Gagnon et al. 31 & 1997 & Canadá & $\begin{array}{l}413 \text { nulíparas, gestação única, } \\
\text { a termo, dilatação cervical } \leq 4 \mathrm{~cm}\end{array}$ & Enfermeira (treinada) & $\begin{array}{l}\text { Permitida presença de } \\
\text { familiares }\end{array}$ \\
\hline Langer et al. 26 & 1998 & México & $\begin{array}{l}724 \text { primíparas com gestação } \\
\text { única, dilatação cervical < } 6 \mathrm{~cm} \text {, } \\
\text { sem indicação de cesariana }\end{array}$ & $\begin{array}{l}\text { Doula (enfermeiras } \\
\text { aposentadas treinadas) }\end{array}$ & Não permitida a presença \\
\hline Madi et al. 32 & 1999 & Botsuana & $\begin{array}{l}109 \text { primiparturientes em } \\
\text { trabalho de parto espontâneo, } \\
\text { sem complicações obstétricas }\end{array}$ & $\begin{array}{l}\text { Parente do sexo feminino } \\
\text { (geralmente a mãe) }\end{array}$ & $\begin{array}{l}\text { Não permitida presença } \\
\text { de familiares. Apenas } \\
\text { durante o estudo, no grupo } \\
\text { de intervenção }\end{array}$ \\
\hline Hodnett et al. 24 & 2002 & $\begin{array}{l}\text { Estados Unidos/ } \\
\text { Canadá }\end{array}$ & $\begin{array}{l}6.915 \text { nulíparas e multíparas, } \\
\text { gestação única ou gemelar, } \\
\text { gestação } \geq 34 \text { semanas }\end{array}$ & Enfermeira (treinada) & Permitida a presença \\
\hline
\end{tabular}

* Estudo não publicado na íntegra, dados extraídos do abstract.

da década de 80 e são parcos, apenas três estavam disponíveis nas principais bases de dados 30. Esta situação parece permanecer, uma vez que na busca bibliográfica para esta revisão somente esses mesmos artigos foram localizados.

Para Gagnon et al. 31, a necessidade de investigar o suporte de enfermeira, nomeado "oneto-one", nos Hospitais da América do Norte, surge pelo fato de que a mesma integra a equipe que assiste a mulher intraparto, possui conhecimentos que conferem um grau de segurança, e habilidades que complementam a dos companheiros, membros da família ou amigos que geralmente já estão presentes. Em seu estudo, o grupo de intervenção, além dos cuidados de rotina intraparto, recebeu suporte contínuo até uma hora após o nascimento, que con- sistia em conforto físico (compressas de calor ou frio, auxílio no banho de chuveiro ou banheira, troca de roupa, ajuda na deambulação, massagem, toque terapêutico e posições de conforto), suporte emocional (tranqüilizar, encorajar, elogiar e distrair), instrução e demonstração de técnicas de relaxamento. Além das atividades de suporte, a enfermeira também atualizava informações para o médico de plantão sobre o progresso do trabalho de parto.

O mais recente ensaio clínico randomizado que avaliou o suporte provido por enfermeiras foi multicêntrico, realizado em 13 hospitais dos Estados Unidos e Canadá. Nesses hospitais, a taxa de cesariana era menor do que $15,0 \%$ e o serviço de analgesia epidural estava disponível nas 24 horas 24 . 
Hodnett et al. 24 identificaram nos ensaios clínicos randomizados, que os provedores de suporte possuíam uma variada experiência, formação e vinculação com o hospital em que desempenhavam esse papel. Dessa forma, analisaram as diferenças entre os provedores serem ou não membros da equipe hospitalar. Dos 15 ensaios clínicos avaliados, em oito o suporte foi provido por membros do hospital (enfermeira, parteira ou estudante de parteira), e em sete por não membros do hospital (doulas, mulheres sem treinamento, educadores perinatais, enfermeiras aposentadas, parente do sexo feminino - mãe). Entretanto, todos os provedores de suporte partilhavam duas características: ser do sexo feminino e possuir experiência com o nascimento, seja por vivência própria ou pela sua formação 4 .

Na maioria dos ensaios clínicos (Tabela 1) não era permitida a presença do companheiro/familiares da parturiente, em apenas três eles estavam presentes para compartilhar a experiência. A presença ou não do companheiro/familiares da parturiente durante o processo do nascimento, neste estudo, pôde ser identificada segundo algumas características: o nível de desenvolvimento do país e o tipo de provedor de suporte. A maioria dos ensaios clínicos em que não era permitida a presença do companheiro ou familiares foi realizada em países em desenvolvimento 21,22,26,27,32 e o suporte era provido por doulas, mulheres leigas sem treinamento e voluntárias da comunidade.

A relação entre a presença ou não do campanheiro/familiares da parturiente e as características hospitalares foi apontada na primeira revisão sistemática publicada na Cochrane Library. Dos 14 ensaios clínicos randomizados, sete foram realizados em hospitais com condições de países tipicamente desenvolvidos (corpo de parteiras/enfermeiras com alto nível de formação, relativa privacidade para a parturiente e altas taxas de intervenção médica), que permitiam a presença do marido/companheiro ou outro membro da família durante o trabalho de parto. Ao contrário, nos estudos realizados em hospitais com condições mais compatíveis com países em desenvolvimento (superlotação, falta de privacidade, profissionais de baixo nível de formação e baixas taxas de intervenção) não era permitida a presença de familiares 15.

\section{Variáveis avaliadas e resultados}

As variáveis avaliadas nos ensaios clínicos e os resultados decorrentes das intervenções estão apresentados na Tabela 2. Observa-se que al- gumas variáveis foram investigadas em um maior número de estudos do que outras. Apenas no ensaio clínico de Kennell \& McGrath et al. 25 não foi possível conhecer todas as variáveis avaliadas, dada a forma de publicação disponível (abstract). A maioria dos estudos avaliou os efeitos do suporte sobre as taxas de cesariana, a duração do trabalho de parto, a analgesia, o uso de ocitocina, a aplicação de fórceps e os resultados neonatais. Uma minoria de ensaios estudou a amniotomia, o uso da monitorização fetal eletrônica contínua e do vácuo extrator, a taxa de parto espontâneo, os traumas perineais, o comportamento/satisfação materna, o aleitamento materno e a depressão pós-parto.

Com relação à cesariana, em quatro estudos 22,23,25,32 a taxa foi significativamente menor no grupo que recebeu suporte, sendo que em dois deles o provedor foi uma doula, nos outros dois foi uma mulher leiga e um familiar do sexo feminino. Nos estudos em que o suporte foi provido por profissional de saúde não houve diferença entre os grupos 24,31,33.

Cabe ressaltar que Kennell \& McGrath 25 analisaram a cesariana relacionada à renda das mulheres e presença dos parceiros. Entre as mulheres de baixa renda $(n=142)$, das quais apenas $43,0 \%$ estavam acompanhadas pelo parceiro, a taxa de cesariana foi similar nos grupos de controle e intervenção (16,4\% vs. 15,9\%). Entretanto, entre as mulheres do grupo de intervenção, que apresentavam nível médio de renda, todas acompanhadas pelo seu parceiro, a taxa foi reduzida de $27,9 \%$ para $14,7 \%$ (controle: $\mathrm{n}=197$, doula: $\mathrm{n}=231 ; \mathrm{x}^{2}=11,2, \mathrm{p}=0,008$ ). Os autores apontam uma possível influência positiva da presença dos parceiros na redução da taxa de cesariana, especialmente em mulheres de média renda.

A metanálise de Scott et al. ${ }^{29}$ classificou os ensaios clínicos de acordo com a característica do suporte provido, ou seja, de forma contínua (durante todo o trabalho de parto/parto) ou intermitente (durante uma parte do trabalho de parto/parto). A redução da taxa de cesariana foi estatisticamente significativa quando prestado de forma contínua $(\mathrm{OR}=0,49$; IC95\%: 0,37-0,65).

A metanálise 28 que avaliou os estudos de Sosa et al. 21, Klaus et al. 22, Kennell et al. 23 e Hofmeyer et al. 27 teve como um dos resultados a diminuição da taxa de cesariana no grupo de mulheres que recebeu o suporte por doula.

A redução da taxa de cesariana também foi identificada nas duas revisões sistemáticas publicadas na Cochrane Library. Hodnett 15 avaliou 14 ensaios clínicos envolvendo mais de 5 
Relação dos ensaios clínicos com as variáveis avaliadas e os resultados.

\begin{tabular}{|c|c|c|c|c|c|c|c|c|c|c|c|c|c|c|c|}
\hline $\begin{array}{l}\text { Ensaios } \\
\text { clínicos/ } \\
\text { ano }\end{array}$ & Ano & $\begin{array}{l}\text { Duração } \\
\text { do traba- } \\
\text { lho de } \\
\text { parto }\end{array}$ & $\begin{array}{l}\text { Ocito- } \\
\text { cina }\end{array}$ & $\begin{array}{l}\text { Amnio- } \\
\text { tomia }\end{array}$ & $\begin{array}{l}\text { Analge- } \\
\text { sia/dro- } \\
\text { gas pa- } \\
\text { ra alívio } \\
\text { da dor }\end{array}$ & $\begin{array}{l}\text { MFE } \\
\text { contí- } \\
\text { nua }\end{array}$ & $\begin{array}{l}\text { Cesari- } \\
\text { ana }\end{array}$ & Fórceps & Vácuo & $\begin{array}{l}\text { Parto } \\
\text { espon- } \\
\text { tâneo }\end{array}$ & $\begin{array}{l}\text { Trauma } \\
\text { peri- } \\
\text { neal }\end{array}$ & $\begin{array}{l}\text { Resul- } \\
\text { tados } \\
\text { neo- } \\
\text { natais }\end{array}$ & $\begin{array}{l}\text { Compor- } \\
\text { tamen- } \\
\text { to/satis- } \\
\text { fação } \\
\text { materna }\end{array}$ & $\begin{array}{l}\text { Aleita- } \\
\text { men- } \\
\text { to ma- } \\
\text { terno }\end{array}$ & $\begin{array}{l}\text { Depres- } \\
\text { são pós- } \\
\text { parto }\end{array}$ \\
\hline $\begin{array}{l}\text { Sosa } \\
\text { et al. } 21\end{array}$ & 1980 & $<\mathrm{GS}$ & & & & & & & & & & & $>\mathrm{GS}$ & & \\
\hline $\begin{array}{l}\text { Klaus } \\
\text { et al. } 22\end{array}$ & 1986 & $<\mathrm{GS}$ & $<\mathrm{GS}$ & & $=* \star$ & & $<\mathrm{GS}$ & $=$ & & & & $\begin{array}{c}\mathrm{GS}< \\
\text { interna- } \\
\text { ção UIN }\end{array}$ & & & \\
\hline $\begin{array}{l}\text { Hodnett } \\
\text { \& Os- } \\
\text { born } 33\end{array}$ & 1989 & $=$ & $=$ & $=$ & $<\mathrm{GS}$ & $=$ & $=$ & $=$ & & & $\begin{array}{l}<\mathrm{GS} \\
\text { (episio- } \\
\text { tomia) }\end{array}$ & & & & \\
\hline $\begin{array}{l}\text { Hofme- } \\
\text { yer et } \\
\text { al. } 27\end{array}$ & 1991 & $=$ & $=$ & $=$ & $=$ & & $=$ & & & & & $=$ & $>\mathrm{GS}$ & $>\mathrm{GS}$ & \\
\hline $\begin{array}{l}\text { Kennell } \\
\text { et al. } 23\end{array}$ & 1991 & $=$ & $=$ & & $<\mathrm{GS}-\mathrm{PE}$ & & $<\mathrm{GS}$ & $<\mathrm{GS}$ & & $=$ & & $=$ & & & \\
\hline $\begin{array}{l}\text { Kennell } \\
\text { \& Mc- } \\
\text { Grath 25* }\end{array}$ & 1993 & & & & & & $<\mathrm{GS}$ & & & & & & & & \\
\hline $\begin{array}{l}\text { Gagnon } \\
\text { et al. } 31\end{array}$ & 1997 & $=$ & $<\mathrm{GS}$ & $=$ & $=$ & & $=$ & $=$ & $=$ & & $=$ & $=$ & & & \\
\hline $\begin{array}{l}\text { Langer } \\
\text { et al. } 26\end{array}$ & 1998 & $<\mathrm{GS}$ & & & $=$ & $<\mathrm{GS}$ & $=$ & $=$ & $=$ & & & $=$ & $=$ & $>\mathrm{GS}$ & \\
\hline $\begin{array}{l}\text { Madi } \\
\text { et al. } 32\end{array}$ & 1999 & & $<\mathrm{GS}$ & $<\mathrm{GS}$ & $<\mathrm{GS}$ & & $<\mathrm{GS}$ & & $<\mathrm{GS}$ & $>\mathrm{GS}$ & $=$ & & & & \\
\hline $\begin{array}{l}\text { Hodnett } \\
\text { et al. } 24\end{array}$ & 2002 & $=$ & $=$ & & $=$ & $=$ & $=$ & $=$ & $=$ & $=$ & $=$ & $=$ & $>\mathrm{GS}$ & $=$ & $=$ \\
\hline
\end{tabular}

* Estudo não publicado na íntegra, dados do abstract, não apresentou todas as variáveis avaliadas;

** O sinal de = representa igualdade entre os grupos de suporte e de controle.

GS = grupo com suporte; PE = parto vaginal espontâneo; UIN = unidade de internação neonatal; MFE = monitorização fetal eletrônica.

mil mulheres (OR = 0,77; IC95\%: 0,64-0,91) e Hodnett et al. 4, 15 ensaios $(\mathrm{n}=12.791$; $\mathrm{RR}=$ 0,90; IC95\%: 0,82-0,99).

O uso de fórceps também foi avaliado pela maioria dos estudos, mas em apenas um deles houve uma redução no seu uso. Nesse estudo o suporte foi provido por doulas 23 .

As variáveis duração do trabalho de parto e uso de analgesia/medicamentos para alívio da dor foram avaliadas em oito ensaios clínicos. Em três deles, ambas as variáveis apresentaram redução no grupo de intervenção, isto é, nas parturientes que receberam suporte. A redução na duração do trabalho de parto foi mais freqüente nos estudos em que o suporte foi provido por mulheres leigas sem treinamento e por doulas. A redução do uso de analgesia ocorreu no estudo de Kennell et al. 23, suporte provido por doulas, mas esta variável foi avaliada apenas nas mulheres que tiveram partos espontâneos. A diminuição do uso de medicamentos para alívio da dor esteve associada ao suporte fornecido por parteira leiga 33 e por familiar do sexo feminino ${ }^{32}$. Cabe ressaltar que Hofmeyer et al. 27 não encontraram diferença estatística entre os grupos em relação à analgesia, mas parturientes do grupo controle solicitaram mais precocemente o seu uso, a dosagem foi maior, com necessidade de doses subseqüentes. Nas revisões publicadas na Cochrane Library 4,15 o uso de medicamento para alívio da dor ou algum tipo de analgesia/anestesia foi menor nas mulheres que receberam suporte.

O uso de ocitocina, avaliado em sete ensaios clínicos, foi reduzido em três deles 22,31,32, mas este resultado não esteve associado a um tipo de provedor de suporte específico; eram mulheres leigas sem treinamento, enfermeiras e parentes do sexo feminino. Entretanto, no estudo de Gagnon et al. 31 os resultados apontaram apenas uma redução de $17,0 \%$ no uso de ocitocina ( $R R=0,83$; IC95\%: 0,67-1,04), sem significância estatística. 
Chamou a atenção o pequeno número de estudos que avaliaram o efeito do suporte sobre o comportamento/satisfação materna (quatro), o aleitamento materno (três) e a depressão pós-parto (um). Essa situação também aparece na revisão sistemática de Hodnett et al. 4 , em que apenas 6 dos 15 ensaios avaliaram os efeitos do suporte sobre a percepção das mulheres quanto à experiência vivida. As que receberam suporte contínuo referiram menor insatisfação ou visão negativa sobre o nascimento $(\mathrm{n}=9.824$; $\mathrm{RR}=0,73$; IC95\%: 0,65-0,83).

Quanto aos resultados neonatais considerados - asfixia intraparto, Apgar do 1o e 5o minutos, tempo de internação, morbidade neonatal (icterícia, taquipnéia, aspiração de mecônio ou sepsis), admissão do recém-nascido em Unidade de Tratamento Intensivo - na maioria dos estudos não se detectou diferenças. Entre seis ensaios 22,23,24,26,27,31 que avaliaram esses aspectos, apenas um 22 obteve uma redução no número de internações na Unidade de Cuidados Neonatais no grupo que recebeu suporte contínuo por mulher leiga. Na primeira revisão sistemática, Hodnett 15 encontrou uma redução no Apgar do 5o minuto menor do que 7 (OR = 0,50; IC95\%: 0,28-0,87). Entretanto, na revisão mais recente 4 essa redução não foi identificada (em sete ensaios, $\mathrm{n}=10.695$; $\mathrm{RR}=0,81$; IC95\%: 0,56-1,16), assim como também não houve uma diminuição das internações dos recémnascidos em unidade de cuidados especiais.

Cabem ainda algumas considerações sobre a avaliação que as metánalises e revisões fazem acerca do suporte no trabalho de parto/parto. A metanálise de Zhang et al. 28 avaliou os ensaios clínicos nos quais o suporte foi provido por mulheres com ou sem treinamento, mas todas denominadas pelos autores como doulas, e apontou como resultado uma redução da duração do trabalho de parto, uso de ocitocina, fórceps e cesarianas; e um aumento no número de partos normais espontâneos, satisfação materna com a experiência e período pós-parto sem intercorrências.

Na metanálise de Scott et al. 29, o suporte, classificado como contínuo ou intermitente, foi provido por mulheres (parteiras leigas, mulheres leigas, parteiras ou estudantes de parteira) denominadas pelo autor como doulas. Em cinco ensaios clínicos o suporte foi contínuo (durante todo o trabalho de parto) e em outros cinco foi intermitente (durante todo o tempo ou parte dele). O suporte contínuo teve um impacto estatisticamente significante na redução da analgesia (OR = 0,64; IC95\%: 0,49-0,85), uso de ocitocina $(\mathrm{OR}=0,29$; IC95\%: 0,20-0,40), fórceps $(\mathrm{OR}=0,43$; IC95\%: 0,28-0,65), taxa de ce- sariana $(\mathrm{OR}=0,49$; IC95\%: 0,37-0,65) e também na duração do trabalho de parto (diferença entre as médias significativas). O suporte intermitente (por parteiras, estudantes de parteira e mulher leiga) não foi significativamente associado a nenhum dos resultados.

As evidências mais recentes sobre o suporte contínuo durante o trabalho de parto/parto foram publicadas na segunda revisão Cochrane 4. A maioria dos ensaios clínicos (13) fizeram parte da revisão anterior 15 , de maneira que algumas características permanecem e outras se modificam de forma sutil. Os efeitos do suporte durante o trabalho de parto estiveram associados com a redução da anestesia/analgesia regional, parto vaginal operatório, parto cesariana, insatisfação ou percepção negativa da parturiente sobre o nascimento. O suporte não foi associado à diminuição do uso de ocitocina, nos escores baixos de Apgar do 5o minuto, na admissão do recém-nascido em unidade de cuidados especiais e na duração do trabalho de parto. Além disso, não influenciou na percepção das puérperas sobre a dor no trabalho de parto ${ }^{4}$.

\section{Discussão e recomendações}

Nos estudos analisados, em geral o suporte durante o trabalho de parto foi realizado por mulheres designadas pelos pesquisadores para desempenhar esse papel, não sendo uma escolha da parturiente. Os companheiros/familiares, que possuem vínculo afetivo com a parturiente, estavam presentes em poucos estudos, quando isto fazia parte da rotina do hospital, mas não no papel de provedor de suporte. Entre todos os estudos, o de Madi et al. 32 foi o único em que o provedor de suporte foi um familiar do sexo feminino, geralmente a mãe da parturiente, mas não por escolha desta.

O suporte realizado por um acompanhante escolhido pela parturiente (marido, companheiro, pai do bebê, membro da familia ou não) não foi avaliado em nenhum dos ensaios clínicos. Fato também evidenciado em recente publicação sobre o tipo de provedor de suporte 30 e na última revisão Cochrane ${ }^{4}$. Existe uma lacuna na literatura sobre os resultados decorrentes desse tipo de suporte.

Os efeitos do suporte à parturiente estiveram mais associados aos resultados maternos, destacando-se redução da taxa de cesariana, seguida pela redução do uso de ocitocina, duração do trabalho de parto, analgesia/medicamentos para alívio da dor e aumento da satisfação materna com a experiência do nascimento. 
Os resultados neonatais parecem não ser diretamente influenciados por esta intervenção, uma vez que eles foram avaliados em quase todos os ensaios clínicos e apenas em um deles se observou redução da internação dos recémnascidos cujas mães receberam suporte.

Em todos os estudos e revisões os resultados são benéficos, não sendo apontado nenhum risco ou prejuízo associado à prática do suporte. Parece que os benefícios são maiores dependendo das características assistenciais dos hospitais, do tipo de provedor e da duração do suporte.

Nos estudos em que os efeitos do suporte sobre a redução das intervenções obstétricas foram mais acentuados, não era permitida a presença do companheiro/familiares da parturiente e as condições hospitalares eram compatíveis com as de países em desenvolvimento (falta de privacidade da parturiente, superlotação, entre outros). Esses aspectos influenciam na forma como o profissional de saúde atua no acompanhamento do trabalho de parto e parto, assim como na atitude das mulheres frente à vivência. Além das variáveis avaliadas, que foram amplamente discutidas nos diferentes estudos, alguns autores destacaram que aspectos comportamentais e culturais podem ter influenciado nos resultados, embora não tenham sido medidos, devido à sua subjetividade. Langer et al. 26 consideram que o limitado impacto do suporte sobre as intervenções pode ser decorrente da realização dos procedimentos hospitalares de forma rotineira, do contexto cultural das mulheres, da pequena duração do suporte e do perfil das doulas. Hodnett et al. 4 destacam que o suporte é mais efetivo quando inicia precocemente (no começo do trabalho de parto) e se a instituição não dispõe de anestesia epidural de rotina.

Quanto ao tipo de provedor, em alguns estudos, todas as mulheres, independente de terem recebido ou não treinamento, possuírem ou não algum tipo de formação na área da saúde, foram denominadas como doulas. Percebese que há certa ambigüidade quanto ao papel e status desse personagem no acompanhamento do trabalho de parto/parto. São consideradas doulas mulheres voluntárias ou pagas para oferecer o suporte, com ou sem experiência técnica na área da saúde, que desempenham atividades estritamente relacionadas às necessidades de conforto físico/emocional da parturiente, ou que também atuam na transmissão de instruções médicas.

A vinculação da doula com o serviço de saúde difere de acordo com a realidade assistencial, de modo que, algumas vezes, faz parte do serviço voluntário do hospital, mas na maioria das vezes os custos do seu serviço são arcados pela parturiente $4,34,35$.

Recente levantamento nacional sobre doulas como "childbirth paraprofessionals", realizado nos Estados Unidos, mostra que elas são remuneradas, pelas clientes, para desempenharem esse papel. A maioria delas possui treinamento ou experiência relacionada com a saúde, ou seja, eram enfermeiras, parteiras, massagistas ou tinham algum treinamento em preparação para o nascimento. Um número significativo delas não se sentem apoiadas e respeitadas pelos médicos, enfermeiras obstetras, outros clínicos e administradores dos serviços de saúde 36 .

Hodnett et al. 4 ressaltam que o maior achado da última revisão sistemática é de que os efeitos do suporte contínuo intraparto estão associados a maiores benefícios quando o provedor não é membro do corpo de funcionários do hospital. Isto ficou evidenciado nos resultados dos ensaios clínicos em que o suporte foi provido por parteiras e enfermeiras $24,31,33$, treinadas sob o modelo médico, com mais ênfase na técnica do que no manejo emocional e social do trabalho de parto 29.

Nos estudos realizados em hospitais de países desenvolvidos, em que a família está presente no processo do nascimento, o provedor de suporte foi um profissional de saúde 24,31,33. Entretanto, os resultados foram similares para os grupos de intervenção e controle, o que pode estar relacionado ao fato de que em ambos os grupos as mulheres também receberam apoio do companheiro/familiares. Para Hodnett et al. 4 , o efeito do suporte parece ser mais forte nos locais em que não é permitida a presença do companheiro e familiares, que podem prover algum apoio.

Em contrapartida, a maioria dos ensaios clínicos realizados em países em desenvolvimento apresentou duas características marcantes: não permitir a presença do companheiro ou de outro membro da família e o suporte ser provido por doula e mulher leiga. Isto pode estar associado a questões culturais do país ou estruturais do hospital. Além disso, pode ser também uma forma de preservar a dinâmica do trabalho hospitalar, uma vez que o suporte provido por mulheres, de alguma forma vinculadas ao serviço ou à parturiente, não requer mudanças para manter a privacidade das parturientes em pré-parto coletivo.

Atualmente existe um movimento mundial para a humanização do nascimento, composto por grupos sociais organizados, em diferentes países, que têm se mobilizado para que ocor- 
ram mudanças em diversos aspectos da assistência obstétrica, entre eles a adoção de práticas baseadas em evidências, que inclui o suporte durante o trabalho de parto e parto 37 .

No Brasil, o Ministério da Saúde considera as recomendações da OMS para a assistência ao parto como referência para as práticas realizadas nas maternidades vinculadas ao SUS. Uma das recomendações é o apoio à mulher durante o trabalho de parto/parto 16 .

Alguns anos antes dessa recomendação, algumas maternidades e casas de parto já permitiam a presença do acompanhante de escolha da mulher (companheiro, mãe, irmã, amiga ou outro). Essas experiências têm sido apresentadas e publicadas como relatos, estudos descritivos e qualitativos, nos quais esse tipo de acompanhante é apontado como um componente que contribui para a satisfação das mulheres com a experiência do nascimento 17,18,34,38. Entretanto, nos dias atuais, a maioria das parturientes ainda não possui um acompanhante para lhe dar apoio durante todo o processo do nascimento.

Apesar do recente sancionamento da Lei $n$. 11.108 11, em abril de 2005, que garante às parturientes o direito à presença de acompanhante durante o trabalho de parto, parto e pós-parto imediato, no âmbito do SUS, tem-se um vasto caminho a ser percorrido para a sua implementação. A presença desses novos sujeitos, acompanhantes de trabalho de parto e parto, pode provocar reações positivas e negativas nos profissionais, sendo que alguns são mais receptivos e estimulam a sua participação, e outros se sentem invadidos e questionados 12,13,18.

Estudos qualitativos mostram que os profissionais possuem uma rejeição inicial, que pode ser atribuída ao preconceito e medo de se sentirem ameaçados e questionados sobre a conduta profissional. No entanto, após terem a experiência de prestar assistência com acompanhante presente, passam a considerá-la positiva, inclusive a diminuição de alguns problemas é atribuída à presença do acompanhante 12,13.

Diante das evidências de que o suporte durante o trabalho de parto gera resultados maternos benéficos, a atual legislação cria a possibilidade de que as parturientes recebam alguma forma de suporte. Aquelas que não possuem uma pessoa de seu meio familiar e/ou afetivo com disponibilidade para acompanhála durante o trabalho de parto poderiam, inclusive, ser acompanhadas por doulas voluntárias, que já têm se mostrado úteis para prover suporte para essas mulheres 34,35 .

A presença do acompanhante, escolhido pela parturiente, considerada pela maioria dos profissionais como um "problema”, pode se transformar numa oportunidade para que o serviço também se beneficie dessa presença. Para tanto, os profissionais devem interagir com esse acompanhante e fornecer orientações necessárias, no momento da internação da parturiente, para que essa pessoa desempenhe o papel de provedor de suporte. Esse acompanhante precisa ser visto como alguém que está vivenciando um momento especial, logo ele também precisa ser acolhido no contexto assistencial em que estiver inserido. Isto, por certo, produzirá um sentimento de confiança e reconhecimento do seu papel, que refletirá positivamente no desenvolvimento de suas atividades de conforto físico e emocional.

As mudanças no contexto assistencial também requerem que as evidências disponíveis sobre o suporte durante o trabalho de parto sejam conhecidas e debatidas nas instituições de saúde e nos foros profissionais, de maneira a viabilizar e disseminar a boa prática e seus benefícios, para que efetivamente se alcance esse aspecto da humanização do nascimento.

Além disso, a determinação legal ora sancionada no Brasil, de que a mulher pode ter um acompanhante de sua escolha durante o trabalho de parto e parto, abre grandes possibilidades de pesquisa para esclarecer e aprofundar as questões aqui discutidas, especialmente em que circunstâncias assistenciais (características do local do nascimento, rotinas de manejo do trabalho de parto/parto e profissionais que assistem a parturiente) o suporte provido por acompanhante escolhido pela parturiente pode ser mais benéfico sobre resultados maternos e perinatais e na satisfação da mulher com a experiência do nascimento. Também se abre a oportunidade de avaliar em nosso meio, de forma mais consistente, os efeitos do companheiro/familiar ou amiga como provedor de suporte, em relação a outros (doulas ou profissionais de saúde), e quais as informações devem ser fornecidas ao acompanhante de escolha da parturiente para que possa desempenhar o papel de suporte. A produção desse conhecimento, a partir da realidade brasileira, poderá fornecer subsídios que colaborem para a implementação da legislação em vigor. 


\section{Resumo}

Os efeitos do suporte à mulher durante o trabalho de parto/parto por profissionais de saúde, mulheres leigas e doulas, sobre os resultados maternos e neonatais têm sido avaliados em vários ensaios clínicos randomizados, metanálises e revisões sistemáticas. Este artigo apresenta a revisão desses estudos, enfocando as principais características, o provedor de suporte, a simultaneidade na presença ou não do companheiro/familiares da parturiente durante o trabalho de parto e parto, e os resultados obtidos. Foram incluídos os estudos publicados entre os anos de 1980 e 2004, que contemplam explicitamente os aspectos avaliados. De maneira geral, os resultados do suporte são favoráveis, destacando-se redução da taxa de cesarianas, da analgesia/medicamentos para alívio da dor, da duração do trabalho de parto, da utilização de ocitocina e produzindo aumento na satisfação materna com a experiência vivida. Quando o provedor de suporte não é um profissional de saúde, os benefícios têm sido mais acentuados. Os estudos disponíveis não avaliam o acompanhante escolhido pela parturiente como um provedor de suporte, o que constitui lacuna de conhecimento a ser preenchida.

Trabalho de Parto; Parto; Assistência Perinatal

\section{Colaboradores}

O. M. Brüggemann participou de todas as etapas de desenvolvimento do artigo. M. A. Parpinelli e M. J. D. Osis participaram da idealização do artigo, análise e revisão do texto.

\section{Agradecimentos}

O presente trabalho foi realizado com o apoio da Coordenação de Aperfeiçoamento de Pessoal de Nível Superior (CAPES).

\section{Referências}

1. Tanaka ACA. Maternidade: dilema entre nascimento e morte. São Paulo: Editora Hucitec/Rio de Janeiro: ABRASCO; 1995.

2. Kitzinger S. Mães: um estudo antropológico da maternidade. Lisboa: Editorial Presença; 1996.

3. Diniz CSG. Entre a técnica e os direitos humanos: possibilidades e limites da humanização da assistência ao parto [Tese de Doutorado]. São Paulo: Departamento de Medicina Preventiva, Universidade de São Paulo; 2001.

4. Hodnett ED, Gates S, Hofmeyer GJ, Sakala C. Continuous support for women during childbirth (Cochrane Review). In: The Cochrane Library, Issue 2, 2005. Oxford: Update Software.

5. Hotimsky SN, Alvarenga AT. A definição do acompanhante no parto: uma questão ideológica? Estudos Feministas 2002; 2:461-81.

6. Storti JPL. O papel do acompanhante no trabalho de parto e parto: expectativas e vivências do casal [Dissertação de Mestrado]. Ribeirão Preto: Escola de Enfermagem de Ribeirão Preto, Universidade de São Paulo; 2004.

7. Pinto CMSP, Basile ALO, Silva SF, Hoga, LAK. O acompanhante no parto: atividades e avaliação da experiência. REME Rev Min Enferm 2003; 7:41-7.

8. Anonymous. Appropriate technology for birth. Lancet 1985; 2:436-7.

9. Rede Nacional Feminista de Saúde, Direitos Sexuais e Reprodutivos. Dossiê de humanização do parto. São Paulo: Rede Nacional Feminista de Saúde, Direitos Sexuais e Reprodutivos; 2002.

10. Salvati I. Parto humanizado. http://www.ideli. com.br/artigosler (acessado em 05/Mai/2004).

11. Brasil. Lei n. 11.108. Altera a Lei n. 8.080, de 19 de setembro de 1990, para garantir às parturientes o direito à presença de acompanhante durante o trabalho de parto, parto e pós-parto imediato, no âmbito do Sistema Único de Saúde - SUS. Diário Oficial da União 2005; 8 abr.

12. Ratto KMN. É possível humanizar a assistência ao parto? Avaliação de dois anos da Maternidade Leila Diniz. Saúde em Foco 2001; 21:115-35.

13. Florentino LC. A participação do acompanhante no processo de nascimento numa perspectiva de humanização [Tese de Doutorado]. São Paulo: Escola de Enfermagem, Universidade de São Paulo; 2003.

14. Enkin M, Keirse MJC, Neilson JP, Crowther C, Duley L, Hodnett E, et al. Guia para atenção efetiva na gravidez e no parto. Rio de Janeiro: Guanabara Koogan; 2005.

15. Hodnett ED. Caregiver support for women during childbirth. Cochrane Database Syst Rev 2002; 1:CD000199.

16. Organização Mundial da Saúde. Assistência ao parto normal: um guia prático. Genebra: Organização Mundial da Saúde; 1996.

17. Domingues RMSM. Acompanhantes familiares na assistência ao parto normal: a experiência da Maternidade Leila Diniz [Dissertação de Mestrado]. Rio de Janeiro: Escola Nacional de Saúde Pública, Fundação Oswaldo Cruz; 2002.

18. Tornquist CS. Paradoxos da humanização em uma 
maternidade no Brasil. Cad Saúde Pública 2003; 19 Suppl 2:419-27.

19. Drummond JP, Silva E, Coutinho M. Medicina baseada em evidências. São Paulo: Editora Atheneu; 2002.

20. Carr KC. Developing and evidence-based practice protocol: implications for midwifery practice. J Midwifery Womens Health 2000; 45:544-50.

21. Sosa R, Kennell J, Klaus M, Robertson S, Urrutia J. The effect of a supportive companion on perinatal problems, length of labor, and mother-infant interaction. N Engl J Med 1980; 303:597-600.

22. Klaus MH, Kennell JH, Robertson S, Sosa R. The effects of social support during parturition on maternal and infant mordidity. BMJ 1986; 293: 585-7.

23. Kennell J, Klaus M, Mcgrath S, Robertson S, Hinkley C. Continuous emotional support during labor in a US hospital. JAMA 1991; 265:2197-201.

24. Hodnett ED, Lowe NK, Hannah ME, Willan AR, Stevens B, Weston, JA, et al. Effectiveness of nurses as providers of birth labor support in North American hospitals: a randomized controlled trial. JAMA 2002; 288:1373-81.

25. Kennell J, McGrath S. Labor support by a doula plus father $v s$. father alone for middle-income couples - the effect on perinatal outcomes. J Dev Behav Pediatr 1993; 14:277.

26. Langer A, Campero L, Garcia C, Reynoso S. Effects of psychosocial support during labour and childbirth on breastfeeding, medical interventions, and mothers' wellbeing in a Mexican public hospital: a randomised clinical trial. Br J Obstet Gynaecol 1998; 105:1056-63.

27. Hofmeyer GJ, Nikodem VC, Wolman WL, Chalmers BE, Kramer T. Companionship to modify the clinical birth environment: effects on progress and perceptions of labour, and breastfeeding. $\mathrm{Br} \mathrm{J} \mathrm{Ob-}$ stet Gynaecol 1991; 98:756-64.
28. Zhang J, Bernasko JW, Fahs M, Hatch MC. Continuous labor support from attendant for primiparous women: a meta-analysis. Obstet Gynecol 1996; 4:739-44.

29. Scott KD, Berkowitz G, Klaus M. A comparison of intermittent and continuous support during labor: a meta-analysis. Am J Obstet Gynecol 1999; 180:1054-9.

30. Rosen P. Supporting women in labor: analysis of different types of caregivers. J Midwifery Womens Health 2004; 49:24-31.

31. Gagnon A, Waghorn K, Covell C. A randomized trial of one-to-one nurse support of women in labor. Birth 1997; 24:71-7.

32. Madi BC, Sandall J, Bennett R, MacLeod C. Effects of female relative support in labor: a randomized controlled trial. Obstet Gynecol 1999; 54:627-8.

33. Hodnett ED, Osborn RW. Effects of continuous intrapartum professional support on childbirth outcomes. Res Nurs Health 1989; 12:289-97.

34. Leão MRC, Bastos MARB. Doulas apoiando mulheres durante o trabalho de parto: experiência do Hospital Sofia Feldman. Rev Latinoam Enfermagem 2001; 9:90-4.

35. Silva AVR. O suporte à parturiente: a dimensão interpessoal no contexto da assistência ao parto [Tese de Doutorado]. São Paulo: Faculdade de Saúde Pública, Universidade de São Paulo; 2004.

36. Lantz PM, Low LK, Varkey S, Watson RL. Doulas as childbirth paraprofessionals: results from a national survey. Womens Health Issues 2005; 15: 109-16.

37. Goer H. Humanizing birth: a global grassroots movement. Birth 2004; 31:308-14.

38. Santos OMB, Siebert ERC. The humanization of birth experience at the University of Santa Catarina maternity hospital. Int J Gynecol Obstet 2001; 48 Suppl 1:23-52.

Recebido em 23/Dez/2004

Versão final reapresentada em $02 / J u n / 2005$

Aprovado em 03/Jun/2005 